\title{
The effect of the viewing distance of stimulus on SSVEP response for use in Brain Computer Interfaces
}

\author{
Chi-Hsu Wu, Heba Lakany \\ Department of Biomedical Engineering \\ University of Strathclyde \\ Glasgow, UK \\ \{chihsu.wu, heba.lakany\}@strath.ac.uk
}

\begin{abstract}
Most visual stimulators used in steady state visual evoked potential (SSVEP) based brain-computer interface (BCI) to elicit SSVEP response are set close to the BCI system limiting the portability and the applications of the system. Our aim is to develop a portable SSVEP based BCI adaptable to the change of the viewing distance of the stimulator which will allow users to operate the BCI without being restricted to a particular area. This study (1) investigates the impact of the distance between the user and the visual stimulator on the SSVEP response and (2) develops a portable visual stimulator that would adapt to change of distance. The finding suggests that a distance adaptable SSVEP based BCI is achievable by changing the intensity of visual stimulator to correspond to the change of the viewing distance. It is hoped that this work can help SSVEP BCI design and broaden its potential applications and users.
\end{abstract}

Keywords: Brain-computer interface (BCI), Steady state visual evoked potential (SSVEP), Electroencephalography (EEG)

\section{INTRODUCTION}

Brain-computer interfaces (BCI) provide an alternative communication channel which does not depend on normal motor output of the nervous system between people with neuromuscular impairment and their external environment [1][2][3]. BCI requires a brain signal associated with a task from the user in order to interpret the user's intent. BCIs can use invasive or non-invasive methods to obtain the brain signal. Non-invasive electroencephalography (EEG) is the most common and preferred method to acquire EEG signal due to its low risk, low cost and easy setup [4][5]. Steady state visual evoked potential (SSVEP) is the brain response to the repetitive visual stimulus of frequencies over $6 \mathrm{~Hz}$ and can be recorded in EEG.

BCIs can be classified as an endogenous or exogenous system, depending on the brain signals used as the input [1]. The brain signals, such as mu rhythms, sensor motor rhythms, slow cortical potentials, are used in endogenous system [1]. The exogenous system uses the signals such as visual evoked potentials (VEP), steady state visual evoked potentials and P300 [1]. These BCI paradigms have successfully conveying the brain signals into the control commands [6]. Compared to other BCI paradigms, SSVEP based BCI has the advantages of high accuracy rate, speed, scalability and less training time [2][7][8]. SSVEP based BCI is also classified as an asynchronous system, i.e. a self-paced system, which does not

This project is funded by EPSRC (Engineering and Physical Sciences Research Council). require a cue to operate it [9] and users can operate the $\mathrm{BCI}$ continuously.

SSVEP based BCIs require the visual stimulators which can be presented by the flickering graphic patterns in the monitors or presented by the flash lights, e.g. light emitting diodes (LEDs) [2], in order to elicit SSVEP response. Currently, SSVEP based BCI applications such as a virtual phone dial pad [10], prosthesis control [11], QWERTY LED keyboard [12] and spelling system [13][14] etc. have been developed. These visual stimulators are normally set close to the users in the distance less than $100 \mathrm{~cm}$.

. This study aims to develop a SSVEP based BCI which allows users to operate the system at any given distance $(<=350 \mathrm{~cm})$. The distance-adaptable feature can take advantage of self-paced system to enhance the flexibility and increase the potential applications and users. SSVEP response is affected by stimulation type, stimulating frequency and stimulus color [2]. In the stimulation type of the light, SSVEP response is sensitive to modulation depth which is related to the stimulus intensity [2] [15]. The intensity of the light is proportional to the inverse square of the distance from the light. This study starts by investigating the impact of the viewing distance between the visual stimulator and the users on SSVEP response. This study uses LEDs as the visual stimulator which is easy to integrate into most environments [2].

The rest of the paper is organized as follows: Section II presents data acquisition experiment setup, protocol, and data analyzed methods. In section III, the results are discussed. Finally, the conclusion and future work are presented in Section IV.

\section{METHODS}

\section{A. Data Acquisition}

Two subjects participated in the experiment. Subjects were seated on a comfortable chair in a dim room. Surface EEG is recorded using a 128 channels EEG cap with a 10-20 international system montage. SSVEP can be recorded most significantly at the channels over the visual cortex [10][15]. Eleven channels over visual cortex were selected as signal channels. $\mathrm{Cz}$ was chosen as the ground and $\mathrm{Fz}$ was chosen as the reference channel. Fig. 1 shows the channel selection. 


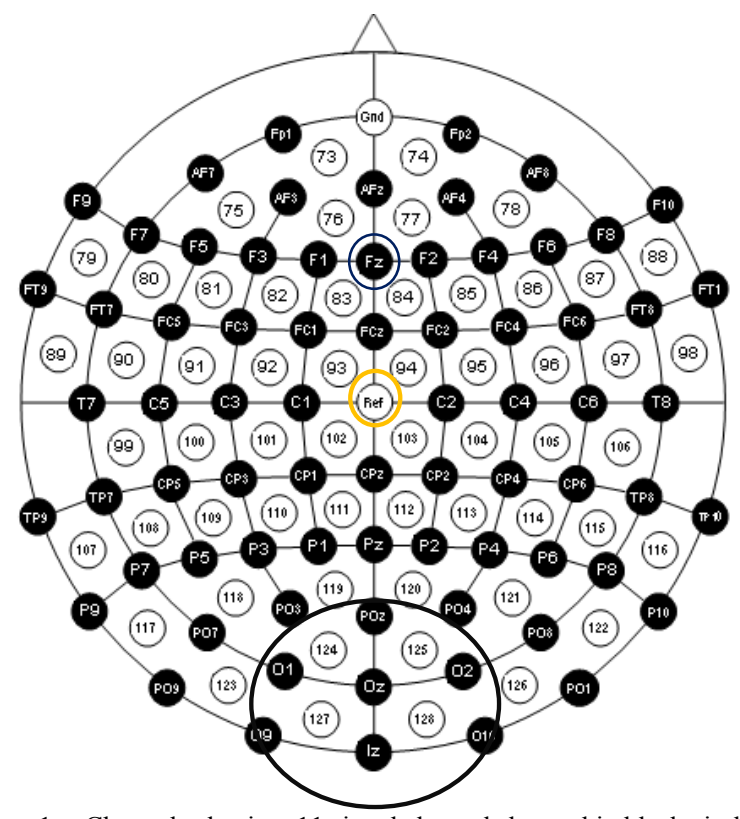

Figure 1. Channel selection. 11 signal channels located in black circle. $\mathrm{Cz}$ as ground located in yellow circle. Reference channel Fz located in blue circle. Channel location is from www.easycap.de.

EEG abrasive skin prepping gel (Nuprep Gel) and EEG conductive gel (Electro-Gel) were applied to the electrode sites to eliminate the dead skin and reduce the impedance between scalp and electrodes. The impedance was kept under $5 \mathrm{k} \Omega$ throughout the entire experiment. The EEG acquisition hardware and software were SynAmps ${ }^{2}$ (amplifier) and NeuroScan 4.5 (recording software). The EEG sampling frequency was $2,000 \mathrm{~Hz}$.

\section{B. Visual Stimulator and Experiment Protocol}

The visual stimulator has one red LED (OSRAM ${ }^{\mathrm{TM}}$, LR G6SP-CADB-1-1, 7100mcd for higher intensity, or LS E63BBBCB-1-1, 2525mcd for lower intensity. Both LEDs are surface mount devices.) LED is driven by a square wave which is generated by the microcontroller (MICROCHIP ${ }^{\mathrm{TM}}$, PIC18F46K20). The microcontroller is programmed to generate four frequencies, 12, 13, 14 and $15 \mathrm{~Hz}$. One of the frequencies is chosen in each experiment and the chosen frequency is used through the whole experiment. The duty cycle of the square wave is $50 \%$.

The experiment records subjects' EEG while they are attending the flickering LED. In one run of the experiment, the visual stimulator with one of four LED intensities was presented to the subject at one of four viewing distances. One complete experiment has 16 runs at the most. Each run has twenty trials. Each trial contains two phases, one resting phase lasting between 5 and 6 seconds randomly and one attending phase lasting 5 seconds. In resting phase, the LED is turned off. During the attending phase, the LED is flickering at the selected frequency and the subject is asked to attend the LED. Each experiment takes around 60 minutes. Four viewing distances between the visual stimulator and the subjects are 60 , 150,250 and $350 \mathrm{~cm}$. LED intensities are changed by altering the value of serial resistor with LED, and/or using different LEDs. In each experiment, four different LED intensities are employed.

\section{Data Pre-processing and Analysis}

EEG data was band-pass filtered by $1-50 \mathrm{~Hz}$ before being analyzed. EEG data was extracted from 1 second before stimulus onset to 4 seconds after stimulus onset. Epoch extraction was performed by EEGLAB [17].

EEG was analyzed off-line. Fast Fourier Transform (FFT), Event-Related Spectral Perturbation (ERSP), Inter-Trial Coherence (ITC) and Stimulus-Locked Inter-trace Correlation (SLIC) were used to visualize the SSVEP response. Canonical Correlation Analysis (CCA) was used to classify EEG.

SSVEP is a sinusoidal oscillatory response which has the same fundamental frequency as the frequency of the stimulus and its higher and/or sub-harmonics components [15] [16]. It is a frequency dependent signal. FFT is one of the most commonly used techniques to transfer EEG from the time domain to the frequency domain [10].

EEGLAB toolbox can be used to analyze EEG in terms of ERSP and ITC [17]. ERSP is the measurement of event related power change and ITC is the measurement of synchronization between EEG and the event. In an experiment of $n$ trials, if $F_{k}(f, t)$ is the $k$-th trial of spectrum estimate at time $t$ and frequency $f$, ERSP and ITC can be defined by the following (1) and (2) [17]

$$
\operatorname{ERSP}(f, t)=\frac{1}{n} \sum_{k=1}^{n}\left|F_{k}(f, t)\right|^{2} .
$$

and

$$
\operatorname{ITC}(f, t)=\frac{1}{n} \sum_{k=1}^{n} \frac{F_{k}(f, t)}{\left|F_{k}(f, t)\right|} .
$$

SLIC is an algorithm developed for SSVEP classification based on the fact that visual evoked potential is time-locked to the visual stimulus onsets [8]. Following this concept, intertraces were extracted between two adjacent 'on' of the LED and the inter-trace correlation is computed to examine the effect of the viewing distances to SSVEP response.

Finally, CCA was used to classify EEG into one of the four classes of frequencies. CCA is used to investigate the relationship between two sets of variables [18] [19]. CCA finds a linear transformation of two sets of variables such that the correlation between the two variables is maximized. It has been shown in many studies that CCA can achieve high classification rates on SSVEP based BCI [18][19][20].

\section{RESULTS}

\section{A. Viewing distance impact on SSVEP response}

Fig. 2 and Fig. 3 illustrate the FFT power spectrum of one subject of four viewing distances with (Fig.3) and without (Fig. 2) intensity compensation. The stimulating frequency is $14 \mathrm{~Hz}$ for Fig.2 and Fig. 3. 

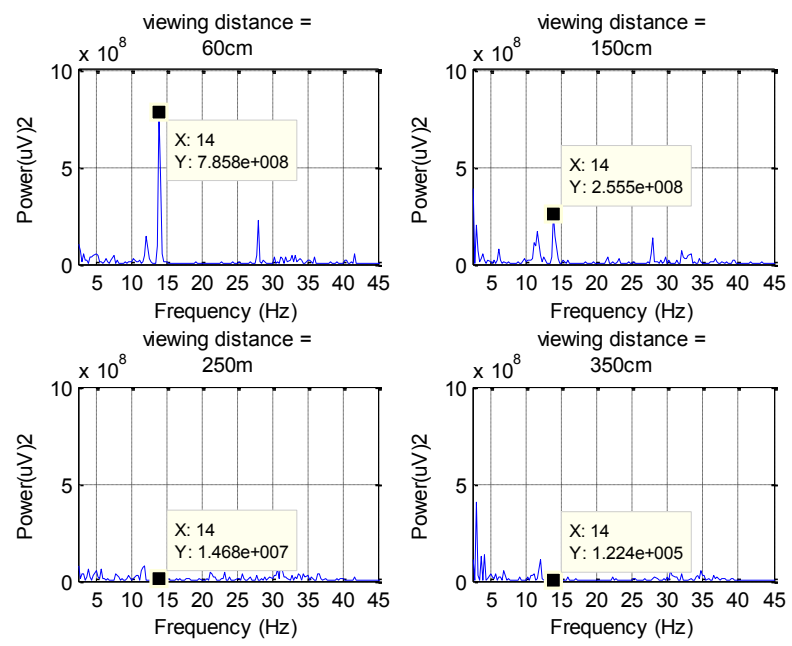

Figure 2. SSVEP response at different viewing distances without intensity compensation. The attending frequency is $14 \mathrm{~Hz}$.

It is clear from Fig. 2 that the SSVEP response becomes weaker and is no longer clear as the viewing distance increases from $60 \mathrm{~cm}$ to $350 \mathrm{~cm}$ without intensity compensation. In Fig. 3, the LED intensities of viewing distances of $150 \mathrm{~cm}, 250 \mathrm{~cm}$ and $350 \mathrm{~cm}$ are stronger than the one of viewing distance of $60 \mathrm{~cm}$. It can be seen from Fig. 3 that with intensity compensation, the longer viewing distance $(150 \mathrm{~cm})$ can elicit stronger SSVEP response than shorter distance $(60 \mathrm{~cm})$. Although SSVEP response also decreases as the viewing distance increases with LED intensity compensation, it is much stronger than the ones without compensation. Fig. 4 and Fig. 5 represent the box plot of SSVEP response and its $p$ value of ANOVA analysis of the data of Fig.2 and Fig 3. $p$ values show that the SSVEP response is significantly different from four different viewing distances with and without LED intensities compensation. Interestingly, longer viewing distance in Fig. 4 shows less variation in SSVEP response than shorter viewing distances.
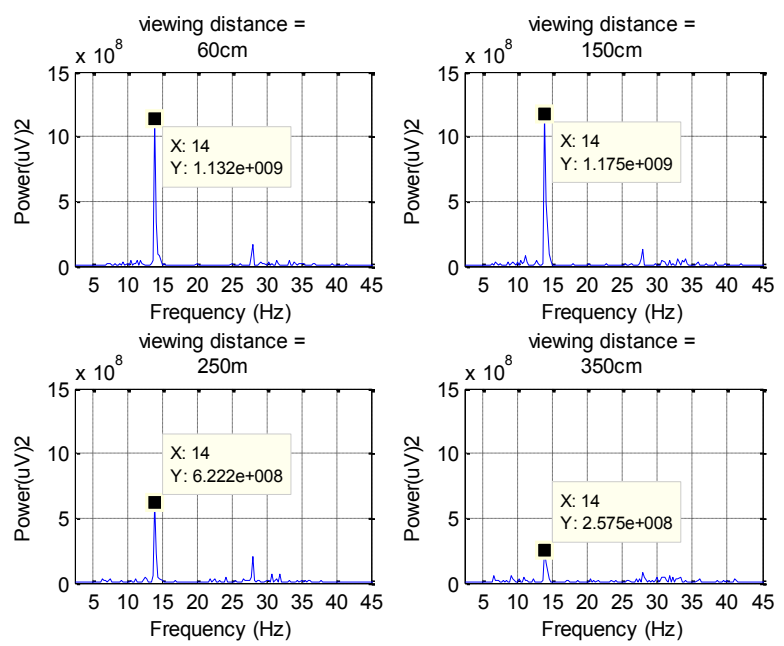

Figure 3. SSVEP response at different viewing distances with intensities compensation. The attending frequency is $14 \mathrm{~Hz}$.
Further post hoc ANOVA analysis shows that in Fig. 4, the mean of SSVEP response of $60 \mathrm{~cm}$ group is significantly different from the means of the groups of the other three distances. The means of the SSVEP response of 150, 250 and $350 \mathrm{~cm}$ groups are not significantly different. The same analysis of Fig. 5 shows that only the mean of SSVEP response of $350 \mathrm{~cm}$ group is significantly different from the ones of groups 60 and $150 \mathrm{~cm}$. No distance groups have means significantly different from the mean of $250 \mathrm{~cm}$ group. This indicates that the intensity compensation can reduce the impact of increasing viewing distance on SSVEP response.

Fig. 6 and 7 depict ERSP and ITC plots of the same LED intensity at different viewing distances, $60 \mathrm{~cm}$ and $350 \mathrm{~cm}$ for Fig. 6 and 7 respectively. The attending frequency is $12 \mathrm{~Hz}$ for both Fig. 6 and 7. Fig. 6 and 7 are drawn by EEGLAB and the bootstrap significance level is set to 0.05 .

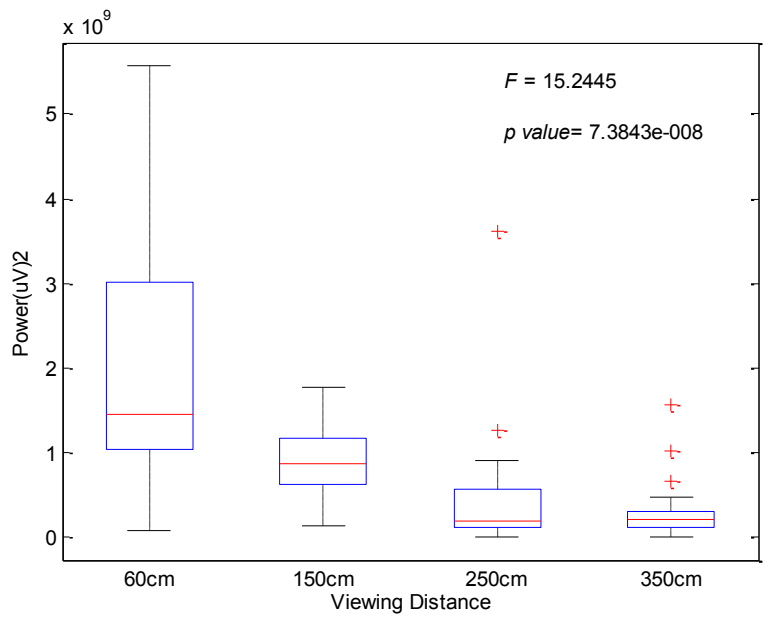

Figure 4. Box plot and ANOVA $p$ value of SSVEP response at different viewing distances with same LED intensity.

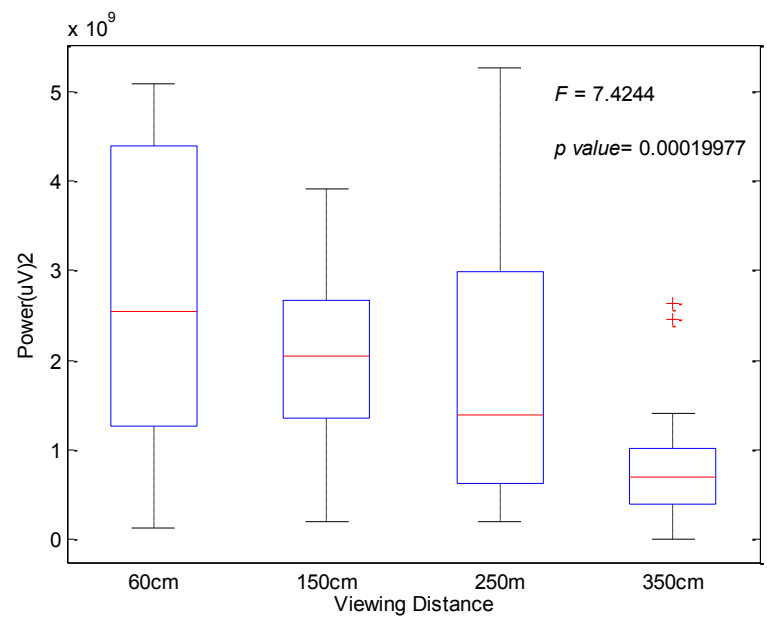

Figure 5. Box plot and ANOVA $p$ value of SSVEP response at different viewing distances with LED intensity compensation. LED intensity of viewing distance $150 \mathrm{~cm}, 250 \mathrm{~cm}$ and $350 \mathrm{~cm}$ is the same and stronger than the one of viewing distance $60 \mathrm{~cm}$. 
Fig. 6 shows that SSVEP response is phase-locked to visual stimulus. There is a clear trace at stimulating frequency $12 \mathrm{~Hz}$ and some cluster at higher harmonic frequencies of the stimulating frequency in ERSP and ITC. When the viewing distance increases, the properties of phase-locked becomes less obvious than the short viewing distance as shown in Fig. 7. There is no clear trace at either the fundamental frequency or higher harmonic frequency. Also the value of ERSP and ITC is less than the short viewing distance.

In Fig. 6 and 7, the thin panel under ERSP image is the envelope of ERSP in time. The blue trace is lowest and the green trace is highest. The green trace in the left panel of ERSP image shows the mean log spectrum. The blue trace in the thin panel under ITC image is ERP average in time. The blue trace in the left panel of ITC image is mean ITC in frequency and the green trace is the ITC significance limits at each frequency [17].

Fig. 8 discloses the result of SLIC analysis at different viewing distances with same LED intensity. The first row of Fig. 8 is the inter-trace EEG data in time domain. The attending frequency of Fig. 8 is $13 \mathrm{~Hz}$. The time length of each inter-trace is 0.07692 second and each trace contains 153 points. When the viewing distance is $60 \mathrm{~cm}$, the mean of EEG traces is sinusoidal-like shape. When the viewing distance increases, the curves of the mean become flatter. The second row of the Fig. 8 is the histogram plot of the inter-trace correlation. There is a clear left skewed at viewing distance $60 \mathrm{~cm}$. As viewing distances increases, the histogram is more like normal distribution with zero mean. The values of the mean of the inter-traces correlation also reflect the same. Fig.9 is the similar result with LED intensity compensation. However, the curves of the mean of the inter-traces are flat and the mean of the inter-trace correlations is very low.

\section{B. Viewing distance impact on frquency}

The impact of viewing distance on different stimulating frequency is also examined. Fig. 10 explains the heat map plot of SSVEP responses of four viewing distances by four different stimulating frequencies. The intensity of four stimulating frequencies is the same.
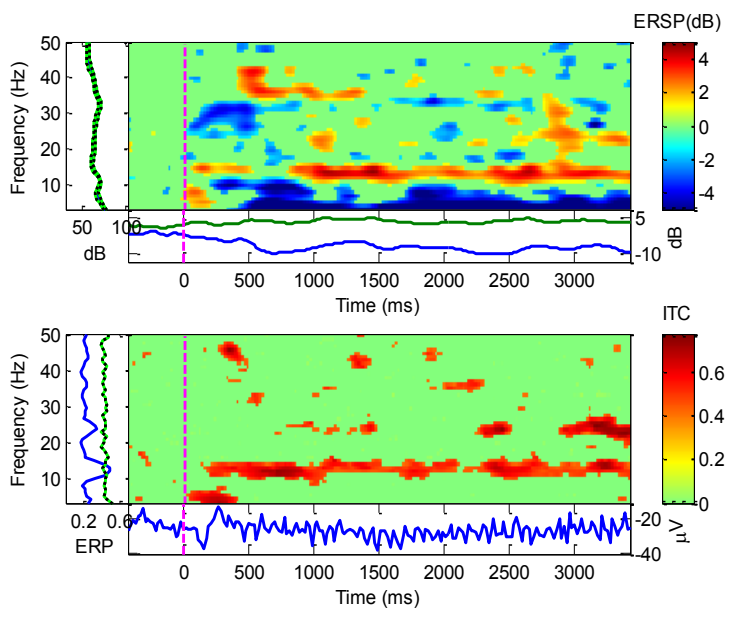

Figure 6. ERSP and ITC plots of viewing distance at $60 \mathrm{~cm}$.
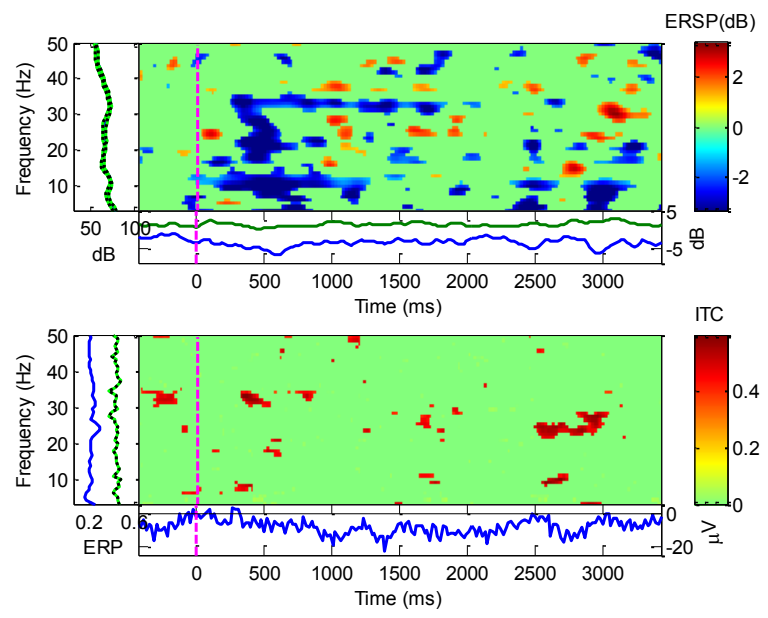

Figure 7. ERSP and ITC plots of viewing distance at $350 \mathrm{~cm}$ with the same LED intensity as Fig. 6.
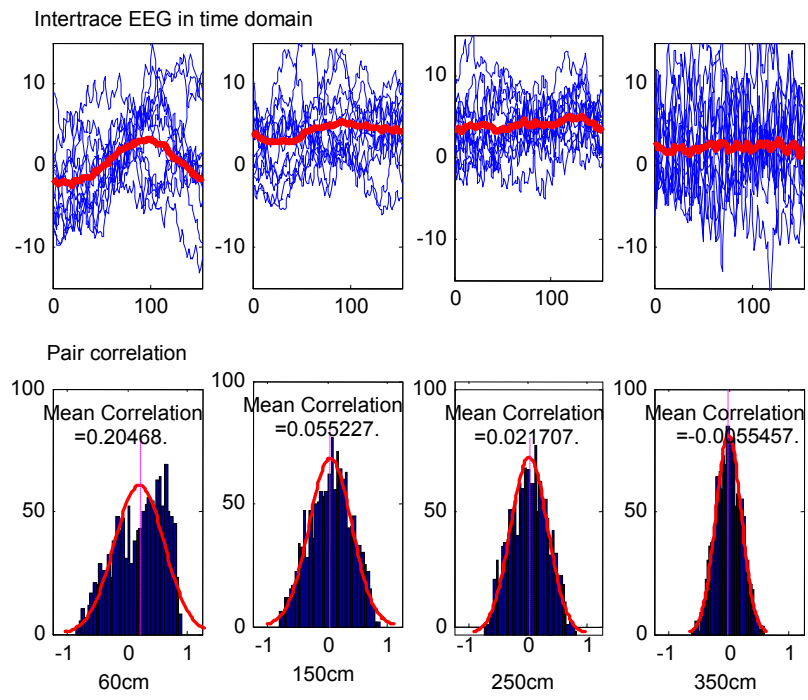

Figure 8. First row is the inter-trace EEG in time domain at different viewing distances. Blue thin line is one single inter-trace and red thick line is the average of all inter-traces. The second row is histogram of the correlation of inter-traces. LED intensity is the same for all viewing distances. The attending frequency is $13 \mathrm{~Hz}$.

There are two significant results to be noted from Fig. 10 . First, SSVEP responses become weaker as the viewing distances increase for all stimulating frequencies. Second, $12 \mathrm{~Hz}$ is less impacted by the increase of the viewing distances. ANOVA is applied to each stimulating frequency for SSVEP response of four viewing distances. The result is listed in Table I. $p$ value shows that the difference of SSVEP responses at four viewing distances of $12 \mathrm{~Hz}$ group is not significant.

TABLE I. ANOVA ANALYSIS OF SSVEP RESPONSE AT DIFFERENT VIEWING DISTANCE FOR EACH STIMULATING FREQUENCY

\begin{tabular}{|c|c|c|c|c|}
\hline & $12 \mathrm{~Hz}$ & $13 \mathrm{~Hz}$ & $14 \mathrm{~Hz}$ & $15 \mathrm{~Hz}$ \\
\hline F value & 2.3427 & 29.6172 & 15.2445 & 54.7196 \\
\hline$p$ value & 0.079762 & $8.68 \mathrm{E}-13$ & $7.38 \mathrm{E}-08$ & $6.005 \mathrm{E}-19$ \\
\hline
\end{tabular}



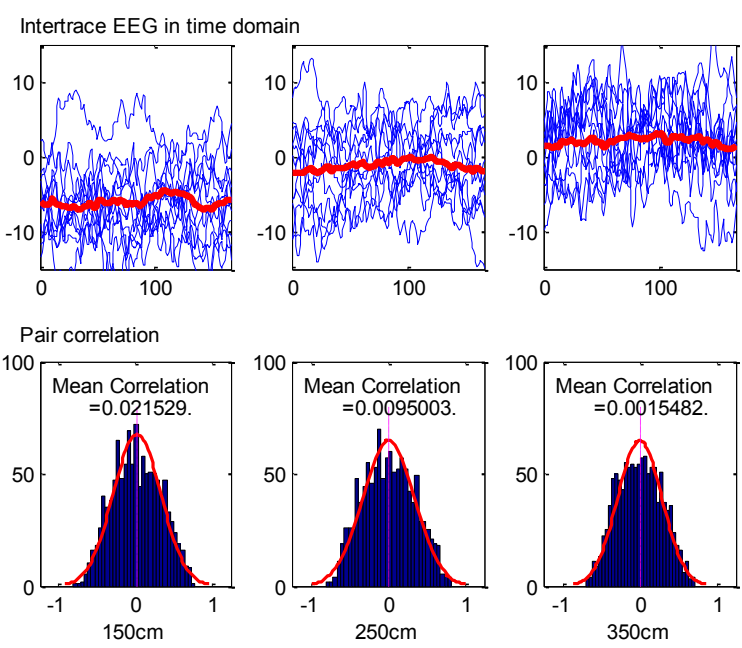

Figure 9. First row shows the inter-trace EEG in time domain at different viewing distances. The blue thin line is one single inter-trace and red thick line is the average of all inter-traces. The second row is histogram of the correlation of inter-traces. In this figure, longer viewing distance has higher LED intensity. The stimulating frequency is $12 \mathrm{~Hz}$.

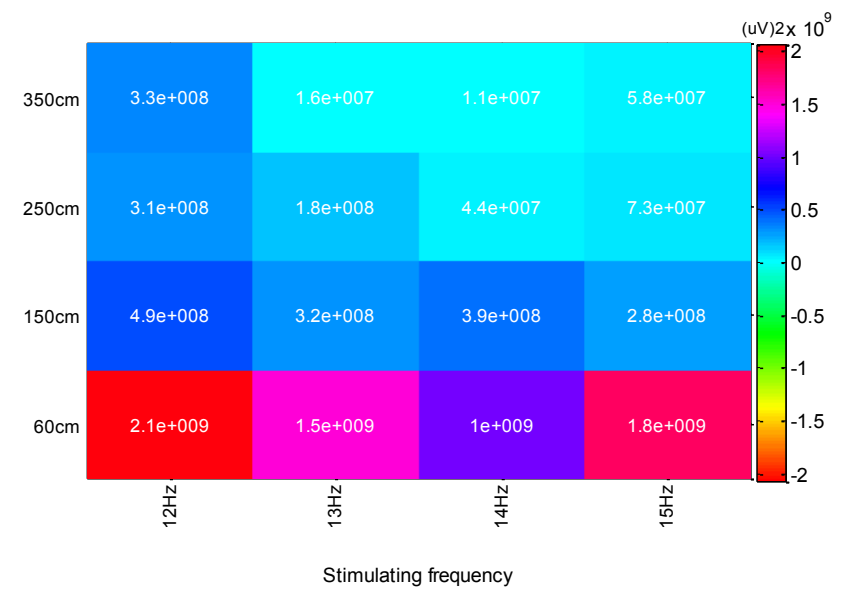

Figure 10. Heat map of SSVEP response of different viewing distances by different stimulating frequencies

\section{Viewing distance impact on classification rate}

After all, BCIs convey the brain signals into commands. Therefore, classification rate is important to BCI application. When applied CCA method to classify EEG, 11 signal channels are used. EEG signal is down sampling by $500 \mathrm{~Hz}$, the time length of each epoch is 2 seconds.

Table II is the classification result of four different viewing distances with the same LED intensity. Table III is the classification result of four different viewing distances with LED intensities increases to response the viewing distance increases. The result of $12 \mathrm{~Hz}$ is not listed in Table III.

From Table II, the classification rates of $12 \mathrm{~Hz}$ are not/less impacted by the increase of the viewing distances. Unlike $12 \mathrm{~Hz}$, the classification rates of the other stimulating frequencies decrease significantly when the viewing distance increases without the intensity compensation. When the viewing distance is over $150 \mathrm{~cm}$, the average classification rate is lower than $20 \%$. Table III shows the classification result of different viewing distances with LED intensity compensation. The classification rates at longer viewing distance improve significantly compared to Table II. The average rate is about $75 \%$.

TABLE II. CONFUSION MATRIX AT DIFFERENT VIEWING DISTANCES WITH SAME LED INTENSITY.

\begin{tabular}{|c|c|c|c|c|c|c|}
\hline $\begin{array}{l}\text { Viewing } \\
\text { distance }\end{array}$ & $\begin{array}{c}\text { Classified } \\
\text { Target }\end{array}$ & $12 \mathrm{~Hz}$ & $13 \mathrm{~Hz}$ & $14 \mathrm{~Hz}$ & $15 \mathrm{~Hz}$ & $\begin{array}{c}\text { Accuracy } \\
\text { (\%) }\end{array}$ \\
\hline \multirow{4}{*}{$60 \mathrm{~cm}$} & $12 \mathrm{~Hz}$ & 20 & 0 & 0 & 0 & $100.00 \%$ \\
\hline & $13 \mathrm{~Hz}$ & 0 & 20 & 0 & 0 & $100.00 \%$ \\
\hline & $14 \mathrm{~Hz}$ & 2 & 0 & 18 & 0 & $90.00 \%$ \\
\hline & $15 \mathrm{~Hz}$ & 1 & 0 & 0 & 19 & $95.00 \%$ \\
\hline \multirow{4}{*}{$150 \mathrm{~cm}$} & $12 \mathrm{~Hz}$ & 20 & 0 & 0 & 0 & $100.00 \%$ \\
\hline & $13 \mathrm{~Hz}$ & 11 & 9 & 0 & 0 & $45.00 \%$ \\
\hline & $14 \mathrm{~Hz}$ & 7 & 2 & 11 & 0 & $55.00 \%$ \\
\hline & $15 \mathrm{~Hz}$ & 11 & 3 & 1 & 5 & $25.00 \%$ \\
\hline \multirow{4}{*}{$250 \mathrm{~cm}$} & $12 \mathrm{~Hz}$ & 19 & 1 & 0 & 0 & $95.00 \%$ \\
\hline & $13 \mathrm{~Hz}$ & 14 & 6 & 0 & 0 & $30.00 \%$ \\
\hline & $14 \mathrm{~Hz}$ & 19 & 0 & 1 & 0 & $5.00 \%$ \\
\hline & $15 \mathrm{~Hz}$ & 17 & 0 & 0 & 3 & $15.00 \%$ \\
\hline \multirow{4}{*}{$350 \mathrm{~cm}$} & $12 \mathrm{~Hz}$ & 18 & 2 & 0 & 0 & $90.00 \%$ \\
\hline & $13 \mathrm{~Hz}$ & 18 & 2 & 0 & 0 & $10.00 \%$ \\
\hline & $14 \mathrm{~Hz}$ & 16 & 2 & 2 & 0 & $10.00 \%$ \\
\hline & $15 \mathrm{~Hz}$ & 15 & 3 & 0 & 2 & $10.00 \%$ \\
\hline
\end{tabular}

TABLE III. CONFUSION MATRIX AT DIFFERENT VIEWING DISTANCES WITH LED INTENSITY COMPENSATION

\begin{tabular}{|c|c|c|c|c|c|c|}
\hline $\begin{array}{l}\text { Viewing } \\
\text { distance }\end{array}$ & $\begin{array}{c}\text { Classified } \\
\text { Target }\end{array}$ & $12 \mathrm{~Hz}$ & $13 \mathrm{~Hz}$ & $14 \mathrm{~Hz}$ & $15 \mathrm{~Hz}$ & $\begin{array}{c}\text { Accuracy } \\
\text { (\%) }\end{array}$ \\
\hline \multirow{3}{*}{$150 \mathrm{~cm}$} & $13 \mathrm{~Hz}$ & 0 & 20 & 0 & 0 & $100.00 \%$ \\
\hline & $14 \mathrm{~Hz}$ & 2 & 0 & 18 & 0 & $90.00 \%$ \\
\hline & $15 \mathrm{~Hz}$ & 1 & 1 & 0 & 18 & $90.00 \%$ \\
\hline \multirow{3}{*}{$250 \mathrm{~cm}$} & $13 \mathrm{~Hz}$ & 5 & 15 & 0 & 0 & $75.00 \%$ \\
\hline & $14 \mathrm{~Hz}$ & 6 & 0 & 14 & 0 & $70.00 \%$ \\
\hline & $15 \mathrm{~Hz}$ & 4 & 1 & 0 & 15 & $75.00 \%$ \\
\hline \multirow{3}{*}{$350 \mathrm{~cm}$} & $13 \mathrm{~Hz}$ & 4 & 16 & 0 & 0 & $80.00 \%$ \\
\hline & $14 \mathrm{~Hz}$ & 8 & 0 & 12 & 0 & $60.00 \%$ \\
\hline & $15 \mathrm{~Hz}$ & 9 & 4 & 0 & 7 & $35.00 \%$ \\
\hline
\end{tabular}

Table IV is the classification rate of different intensities at different viewing distances. I001 to I005 listed in table IV are LED intensities which were tested in the experiment. I001 is the lowest intensity, and I005 is the highest intensity. The classification rate of $12 \mathrm{~Hz}$ is not included in Table IV. In Table IV, the viewing distance $60 \mathrm{~cm}$ has the highest classification rate regardless of the LED intensities used. In general, for a given intensity, the classification rates decrease as the viewing distance increases. For a given viewing distance, the classification rates increase as the intensity (excluding I001) increases.

\section{CONCLUSION AND FUTURE WORK}

The preliminary experiment result shows that at the viewing distance $(60 \mathrm{~cm})$, SSVEP response is prominent and classification rate is high. The result of the experiment confirms that SSVEP response is sensitive to the modulation depth which is highly related to the intensity of the visual stimuli. When the intensity of the LEDs remains the same, the SSVEP becomes weaker and classification rate is lower as a result of the increase 
of viewing distance. Some of the properties of SSVEP, such as time-locked and phase-locked to visual stimuli are also impacted by viewing distance. One survey study [2] reported that red color of the stimulus can elicit strongest SSVEP at the stimulating frequency around $11 \mathrm{~Hz}$ and the response drops dramatically at neighboring frequencies. This might explain why the viewing distance has less impact on $12 \mathrm{~Hz}$.

\section{TABLE IV. ClasSIFICATION RATES AT DIFFERENT VIEWING DISTANCES WITH DIFFERENT LED INTENSITIES.}

\begin{tabular}{c|cccc}
$\begin{array}{c}\text { Distance } \\
\text { Intensity }\end{array}$ & $\mathbf{6 0 c m}$ & $\mathbf{1 5 0} \mathbf{c m}$ & $\mathbf{2 5 0} \mathbf{c m}$ & $\mathbf{3 5 0} \mathbf{c m}$ \\
\hline $\mathbf{I 0 0 1}$ & $97 \%$ & $50 \%$ & $18 \%$ & $10 \%$ \\
$\mathbf{I 0 0 2}$ & $90 \%$ & $8 \%$ & $3 \%$ & $3 \%$ \\
$\mathbf{I 0 0 3}$ & $93 \%$ & $25 \%$ & $10 \%$ & $3 \%$ \\
$\mathbf{I 0 0 4}$ & $98 \%$ & $35 \%$ & $30 \%$ & $8 \%$ \\
$\mathbf{I 0 0 5}$ & $100 \%$ & $90 \%$ & $73 \%$ & $48 \%$ \\
\hline
\end{tabular}

However, the intensity compensation to respond to the change of the viewing distance results in strong SSVEP response and has a higher average classification rate $(\approx 75 \%)$. This finding suggests that a distance adaptable SSVEP based $\mathrm{BCI}$ is achievable by changing the intensity of visual stimulator to respond to the change of the viewing distance.

A practical BCI system should have more than one command. This study so far only investigates the effect of the viewing distances to one target. When applied to BCIs which have more than one command, the viewing distances will impact the viewing angles between two adjacent targets and might cause aliasing. This is what we intend to investigate next.

Meanwhile, there are many wireless EEG recording devices available in the market. The feature of the wireless recording frees the boundaries of use of BCI. To build the visual stimulus on a portable device, such as a pair of the goggles or a visor might take the advantage of the wireless recording device and enhance the usability and portability of BCIs. Such portable visual stimulator requires very short viewing distance. The effect of such short viewing distance to SSVEP is also worth investigating.

\section{ACKNOWLEDGMENT}

The authors would like to thank the subjects who participated in the experiment and Mr. John Maclean who helped in making the visual stimulator.

\section{REFERENCES}

[1] J. R. Wolpaw, N. Birbaumer, W. J. Heetderks, D. J. McFarland, P. H Peckham, G. Schalk, E. Donchin, L. a Quatrano, C. J. Robinson, and T. M. Vaughan, "Brain-computer interface technology: a review of the first international meeting.," IEEE transactions on rehabilitation engineering: a publication of the IEEE Engineering in Medicine and Biology Society, vol. 8, no. 2, pp. 164-73, Jun. 2000.

[2] Danhua Zhu, Jordi Bieger, Gary Garcia Molina, and Ronald M. Aarts, "A Survey of Stimulation Methods Used in SSVEP-Based BCIs," Computational Intelligence and Neuroscience, vol. 2010, doi:10.1155/2010/70235.

[3] Pfurtscheller, Gert, and Scherer, Reinhold, "Brain-computer interfaces used for virtual reality control," Venice, Italy, ICABB, 2010.
[4] Jonathan R. Wolpaw, Niels Birbaumer, Dennis J. McFarland, Gert Pfurtschellere, and Theresa M. Vaughana, "Brain-computer interfaces for communication and control," Clinical Neurophysiology. vol. 113, no. 6, pp. 767-791,2002.

[5] Ivan Volosyak, Diana Valbuena, Tatsiana Malechka, Jan Peuscher, and Axel Gräser, "Brain-computer interface using water-based electrodes," Journal of Neural Engineering. vol. 7, no. 6, doi:10.1088/17412560/7/6/066007,2010.

[6] Ou Bai, Peter Lin, Sherry Vorbach, Mary Kay Floeter, Noriaki Hattori, and Mark Hallett, "A high performance sensorimotor beta rhythm-based brain-computer interface associated with human natural motor behaviour," Journal of Neural Engineering. vol. 5, no. 1, pp. 24-35, 2007.

[7] Pablo Martinez, Hovagim Bakardjian, and Andrzej Cichocki, "Fully online multicommand brain-computer interface with visual neurofeedback using SSVEP paradigm," Computational Intelligence and Neuroscience. vol. 2007, doi:10.1155/2007/94561, 2007.

[8] An Luo, and Thomas J Sullivan, "A user-friendly SSVEP-based braincomputer interface using a time-domain classifier,". Journal of Neural Engineering. vol. 7, no. 2, doi: 10.1088/1741-2560/7/2/026010, 2010

[9] P. F. Diez, V. a Mut, E. M. Avila Perona, and E. Laciar Leber, "Asynchronous BCI control using high-frequency SSVEP.," Journal of neuroengineering and rehabilitation, vol. 8, no. 1, p. 39, Jan. 2011.

[10] M. Cheng, X. Gao, S. Gao, and D. Xu, "Design and implementation of a brain-computer interface with high transfer rates.," IEEE transactions on bio-medical engineering, vol. 49, no. 10, pp. 1181-6, Oct. 2002.

[11] Muller-Putz, G.R.; Pfurtscheller, G., "Control of an Electrical Prosthesis With an SSVEP-Based BCI," Biomedical Engineering, IEEE Transactions on , vol.55, no.1, pp.361-364, Jan. 2008

[12] H.-J. Hwang, J.-H. Lim, Y.-J. Jung, H. Choi, S. W. Lee, and C.-H. Im, "Development of an SSVEP-based BCI spelling system adopting a QWERTY-style LED keyboard.," Journal of neuroscience methods, vol. 208, no. 1, pp. 59-65, Jun. 2012.

[13] Friman, O.; Luth, T.; Volosyak, I.; Graser, A., "Spelling with SteadyState Visual Evoked Potentials," Neural Engineering, 2007. CNE '07. 3rd International IEEE/EMBS Conference on , vol., no., pp.354-357, 2-5 May 2007

[14] I. Volosyak, "SSVEP-based Bremen-BCI interface--boosting information transfer rates.," Journal of neural engineering, vol. 8, no. 3, 036020, Jun. 2011.

[15] S. T. Morgan, J. C. Hansen, and S. a Hillyard, "Selective attention to stimulus location modulates the steady-state visual evoked potential.," Proceedings of the National Academy of Sciences of the United States of America, vol. 93, no. 10, pp. 4770-4, May 1996.

[16] G. R. Müller-Putz, R. Scherer, C. Brauneis, and G. Pfurtscheller, "Steady-state visual evoked potential (SSVEP)-based communication: impact of harmonic frequency components.," Journal of neural engineering, vol. 2, no. 4, pp. 123-30, Dec. 2005.

[17] A. Delorme and S. Makeig, "EEGLAB: an open source toolbox for analysis of single-trial EEG dynamics including independent component analysis.," Journal of neuroscience methods, vol. 134, no. 1, pp. 9-21, Mar. 2004.

[18] Z. Lin, C. Zhang, W. Wu, and X. Gao, "Frequency recognition based on canonical correlation analysis for SSVEP-based BCIs.," IEEE transactions on bio-medical engineering, vol. 54, no. 6 Pt 2, pp. 1172-6, Jun. 2007.

[19] G. Bin, X. Gao, Z. Yan, B. Hong, and S. Gao, "An online multi-channel SSVEP-based brain-computer interface using a canonical correlation analysis method.," Journal of neural engineering, vol. 6, no. 4, p. 046002, Aug. 2009.

[20] F.-B. Vialatte, M. Maurice, J. Dauwels, and A. Cichocki, "Steady-state visually evoked potentials: focus on essential paradigms and future perspectives.," Progress in neurobiology, vol. 90, no. 4, pp. 418-38, Apr. 2010. 